\title{
Actualidad institucional y económica de España en el marco de la Unión Europea
}

\author{
Current Institutional and Economic Developments in Spain within the \\ Framework of the European Union
}

\author{
Beatriz Iñarritu \\ Profesora de la «Deusto Business School» \\ Universidad de Deusto
}

doi: 10.18543/ced-54-2016pp307-325

\begin{abstract}
Sumario: I. Introducción.-II. El Estado de la Integración. 1. Discurso sobre el Estado de la Unión. 2. Tercer rescate de Grecia. 3. Inmigración: planes de acogida a solicitantes de asilo. 4. Reino Unido: nuevos pasos en la negociación con la UE. 5. El Informe de los Cinco Presidentes sobre el futuro de la gobernanza económica europea. 6. Informes sobre la Ampliación de la UE. 7. Comisión: primera aplicación del Mecanismo del Estado de Derecho contra Polonia.-III. Cuestiones generales de la actualidad económica. 1. BCE: Ampliación de la compra masiva de Deuda pública. 2. Acuerdo Trasatlántico UE-EEUU: nuevas propuestas para el arbitraje en materia de inversiones. 3. La Unión de los Mercados de Capitales. 4. Unión Bancaria: Sistema Europeo de Garantía de Depósitos Bancarios. 5. Tribunal de Justicia: nueva sentencia contra la Ley Hipotecaria española. 6. Comisión: dictamen contra las cláusulas suelo en las hipotecas españolas. 7. Competencia: ilegalidad de los pactos fiscales con multinacionales. 8. Telefonía móvil: eliminación de las tarifas de itinerancia en 2017. 9. Tribunal de Justicia anulación de la decisión de la Comisión que declaraba ilegal el Tax Lease del sector naval español.
\end{abstract}

\section{Introducción}

En el segundo semestre de 2015, la actualidad de la Unión Europea se ha centrado en los difíciles retos que se le presentan. Destacan, sin duda, la crisis de los refugiados y los insuficientes resultados conseguidos, y la necesidad de haber tenido que pactar un tercer rescate con Grecia tras el cierre de los bancos del país durante tres semanas.

También debe destacarse la utilización, por vez primera, del mecanismo del Estado de Derecho a comienzos de 2016. Los «excesos» del nuevo gobierno polaco ultraconservador, que cuestionan el respeto de los valores de la Unión, han justificado esta grave alerta. 
Por otra parte, la Unión continúa sus avances en el ámbito financiero, y en particular con el proyecto de la Unión de los Mercados de Capitales y con la definición del Sistema Europeo de Garantía de Depósitos, y sin olvidar la «cruzada» emprendida por la Comisión contra las ventajas fiscales que diversos gobiernos europeos pactan con grandes multinacionales.

In this second semester in 2015, current news about the European Union have been focused on the difficult challenges it is facing. It can be highlighted the refugee crisis and the insufficient results achieved so far, and also the necessity to negotiate and agree a third rescue of Greece after the closure of banks during three weeks.

It could also be underlined that, for the first time, the mechanism of the Rule of Law has been used in the beginning of 2016. The «excesses» made by the new ultra conservative Polish government, that are questioning the respect of the Union's values, have justified this serious alert.

On the other hand, the Union continues progressing in the financial field, being particularly important the project on the Capital Markets Union and the European Deposit Guarantee Scheme, and not forgetting the «crusade» that the Commission has launched against those tax benefits that several European governments agree with big multinationals.

\section{El estado de la integración}

\section{Discurso sobre el Estado de la Unión}

Jean Claude Juncker pronunció en Septiembre su primer discurso sobre el Estado de la UE como presidente de la Comisión ante el pleno del Parlamento Europeo, con el fin de realizar un balance de la situación actual de la Unión Europea y de establecer las prioridades para el futuro.

En su análisis, el político luxemburgués señaló que «no hay suficiente Europa en esta Unión, ni hay suficiente Unión en esta Unión» y pasó revista a los grandes retos a los que se enfrenta la UE actual:

1) La crisis de los refugiados: Juncker señaló que los europeos hemos de recordar que hemos sido, en algún momento, refugiados en éxodos de millones de personas huyendo de las persecuciones religiosas o políticas, la guerra, la dictadura o la opresión.

Realizó un llamamiento a preparar una modificación de calado del tratamiento que se da a las solicitudes de asilo y, también, a la mayor aproximación de las políticas de asilo en la fase posterior a la concesión del estatuto de refugiado. Y señaló, asimismo, que «una solución duradera sólo será posible si se abordan las causas profun- 
das, las razones por las que nos enfrentamos actualmente a esta importante crisis de refugiados». Es por ello que «la política exterior europea debe ser más firme. Ya no podemos permitirnos el lujo de ignorar o de no adoptar una posición unitaria frente a la guerra o la inestabilidad que afecta a las regiones de nuestro entorno».

2) La situación de Grecia: En opinión de Juncker, la crisis económica no ha terminado, puesto que la situación económica y social habla por sí misma: más de 23 millones de personas siguen desempleadas en la Unión Europea y de ellas, más de la mitad ha estado en el paro durante un año o más. La deuda pública de la UE ha superado el 88 $\%$ del PIB de media, y casi el $93 \%$ en la zona euro.

La profundización en la Unión Económica y Monetaria se basará en las conclusiones del Informe de los Cinco Presidentes presentado en Junio, en el que se acordaba una hoja de ruta que debería permitir estabilizar y consolidar la zona euro a más tardar a principios de 2017, para emprender a continuación, sobre la base de una convergencia renovada de las Economías, una reforma más en profundidad y pasar, ahí donde sea posible, de la crisis a la apertura de nuevas perspectivas de crecimiento.

3) La respuesta al Reino Unido: Juncker destacó su disposición a trabajar conjuntamente con el Gobierno británico para alcanzar un acuerdo. Señaló, asimismo, que parte de este pacto consistirá en reconocer que no todos los Estados miembros participan en todas las políticas de la UE e hizo alusión al hecho de que la posición del Reino Unido se define en protocolos especiales, por ejemplo en relación con el euro o la justicia y los asuntos de interior. Y también, señaló que, en contrapartida, las decisiones del Reino Unido no deben impedir a los demás Estados miembros una mayor integración cuando lo consideren oportuno.

4) La posición europea ante Ucrania: El presidente de la Comisión señaló que el apoyo comunitario a este país debe continuar, una vez que ya se le han concedido préstamos por valor de 3.410 millones de euros en tres programas de ayuda macrofinanciera, contribuyendo a alcanzar un acuerdo que garantice el suministro de gas durante el invierno y ofreciendo asesoramiento sobre la reforma del poder judicial.

5) El cambio climático: En palabras de Juncker, el cambio climático también es una de las causas profundas de un nuevo fenómeno migratorio. «Si no actuamos con celeridad, los refugiados climáticos se convertirán en un nuevo reto» concluyó. Es en este sentido que la prioridad de la UE debe ser adoptar un acuerdo mundial sobre el clima que sea ambicioso, sólido y vinculante. 


\section{Tercer rescate de Grecia}

El 5 de Julio el gobierno griego convocó un referéndum para consultar a los ciudadanos griegos sobre la aceptación o no del nuevo plan de rescate que había sido propuesto por las instituciones comunitarias y el FMI y que significaba, por tanto, la decisión sobre la implementación de nuevas políticas de austeridad.

Y a pesar del rechazo mayoritario del pueblo griego, Atenas se encontró con un firme ultimátum de sus socios europeos que le llevó a solicitar, in extremis, un tercer rescate. Y así fue como, en apenas tres días, el 8 de Julio, el primer ministro de Grecia, Alexis Tsipras, aseguraba ante el Parlamento Europeo que su país había presentado formalmente la petición de un nuevo rescate al Mecanismo Europeo de Estabilidad (MEDE) para poder cumplir con las obligaciones de la deuda y asegurar la estabilidad financiera del país.

El Eurogrupo aprobó el 16 de Julio el inicio de las negociaciones con Atenas para acordar esta nueva ayuda dirigida a cubrir las necesidades financieras del país durante los próximos tres años, decisión que coincidió con la adoptada por el Banco Central Europeo (BCE) de aumentar en 900 millones de euros la liquidez de emergencia para los bancos griegos.

El 20 de Julio, y tras tres semanas de cierre, los bancos griegos reabrían sus puertas aunque con muchas restricciones. Pudieron normalizar algunas operaciones en ventanilla, como el pago de cuotas y créditos de todo tipo, aunque las operaciones con el extranjero continuaban estando sujetas a severas limitaciones.

El 14 de Agosto, el Eurogrupo dio luz verde al tercer rescate a Grecia por un valor de 86.000 millones de euros. En su discurso previo ante el Parlamento griego, Tsipras había defendido su decisión de aceptar el programa y las duras exigencias respecto a la subida de impuestos y recortes de gastos sociales, llegando a afirmar que tuvo que elegir «entre seguir vivos o el suicidio». «No lamento la decisión. No estamos exultantes pero tampoco en duelo. Tengo la conciencia limpia porque es lo mejor que pudimos lograr bajo el actual equilibrio de poderes en Europa, bajo las condiciones de asfixia económica y financiera que nos impusieron» señaló.

En la condicionalidad de este nuevo rescate, Grecia se comprometió a aplicar ajustes en materias tan sensibles como las pensiones, el IVA o las privatizaciones, y que previsiblemente significarán en torno al $4 \%$ o $5 \%$ del PIB del país.

La aprobación final del rescate tuvo lugar el 19 de Agosto con la decisión del Consejo de Gobernadores del Mecanismo Europeo de Estabilidad. 
Al día siguiente, 20 de Agosto, Tsipras presentó su dimisión y pidió la celebración de nuevas elecciones, ante la pérdida de la mayoría de su partido Syriza en el Parlamento griego, tras el rechazo de un tercio de sus diputados al tercer rescate. Las elecciones, que se celebraron el 20 de Septiembre, registraron la participación más baja en toda la historia de Grecia y se saldaron con una nueva victoria de Syriza, que obtuvo el 35,35\% de los votos. Tsipras anunció entonces que seguiría gobernando el país con los nacionalistas Griegos Independientes.

\section{Inmigración: planes de acogida a solicitantes de asilo}

Tras el primer plan presentado en Mayo por la Comisión Europea, que planteaba la acogida en los países miembros de 40.000 solicitantes de asilo sirios y eritreos llegados a Grecia e Italia en los últimos meses, Bruselas anunció el 9 de septiembre un nuevo conjunto de medidas que incluía un nuevo mecanismo de reubicación de emergencia para otros 120.000 refugiados en los próximos dos años.

Los Estados miembros apoyaron esta nueva iniciativa el 22 de Septiembre para, en efecto, reubicar a nuevos asilados procedentes de los territorios con más llegadas, Grecia e Italia. Todos los países, incluidos los cuatro que votaron en contra (Hungría, República Checa, Eslovaquia y Rumanía) y el que se abstuvo (Finlandia), deberán acatar la decisión.

En el primer año se distribuirán 66.000 asilados ya asignados entre 23 Estados (todos salvo Italia y Grecia, a los que se pretende aliviar con este acuerdo, y excepto Reino Unido, Irlanda y Dinamarca porque tienen capacidad para no aplicar las políticas europeas de Justicia e Interior).

La redistribución de los 54.000 restantes del segundo año quedaba pendiente a expensas de que en los meses siguientes algún otro Estado miembro pudiera afrontar una «situación de emergencia» que comporte un volumen inusitado de refugiados en sus fronteras (como es el caso de Italia y Grecia) y solicitara un reajuste en el cupo de refugiados.

A pesar de que la Comisión había propuesto que los Estados que no cooperaran en el sistema de reparto por motivos excepcionales aportaran una suma de dinero al presupuesto comunitario para compensar los sobreesfuerzos del resto, finalmente se optó por abandonar esta idea a cambio de limitar la capacidad de los Estados a negarse a acoger refugiados. De esta forma, los Estados solo podrán retrasar un máximo de 12 meses la acogida de hasta el 30\% del cupo asignado, y para ello deberán atravesar «circunstancias excepcionales y alegar motivos debidamente justificados compatibles con los valores fundamentales de la Unión», según señalaba el texto aprobado. 
Distribución de la acogida de refugiados llegados a Italia y Grecia (66.000 refugiados, primer año - Septiembre 2015)

\begin{tabular}{|c|c|c|c|c|}
\hline & $\begin{array}{l}\text { Allocation from } \\
\text { Italy per Member } \\
\text { State } \\
\text { (15 } 600 \text { applicants } \\
\text { relocated) }\end{array}$ & $\begin{array}{c}\text { Allocation from } \\
\text { Greece per Member } \\
\text { State } \\
\text { (50 } 400 \text { applicants } \\
\text { relocated) }\end{array}$ & $\begin{array}{c}\text { Total } \\
\text { Allocations }\end{array}$ & $\%$ \\
\hline Germany & 4.027 & 13.009 & 17.036 & 25,81 \\
\hline France & 3.064 & 9.898 & 12.962 & 19,64 \\
\hline Spain & 1.896 & 6.127 & 8.023 & 12,16 \\
\hline Poland & 1.201 & 3.881 & 5.082 & 7,70 \\
\hline Netherlands & 922 & 2.978 & 3.900 & 5,91 \\
\hline Romania & 585 & 1.890 & 2.475 & 3,75 \\
\hline Belgium & 579 & 1.869 & 2.448 & 3,71 \\
\hline Sweden & 567 & 1.830 & 2.397 & 3,63 \\
\hline Austria & 462 & 1.491 & 1.953 & 2,96 \\
\hline Portugal & 388 & 1.254 & 1.642 & 2,49 \\
\hline Czech Republic & 376 & 1.215 & 1.591 & 2,41 \\
\hline Hungary & 306 & 988 & 1.294 & 1,96 \\
\hline Finland & 304 & 982 & 1.286 & 1,95 \\
\hline Bulgaria & 201 & 651 & 852 & 1,29 \\
\hline Slovakia & 190 & 612 & 802 & 1,22 \\
\hline Croatia & 134 & 434 & 568 & 0,86 \\
\hline Lithuania & 98 & 318 & 416 & 0,63 \\
\hline Slovenia & 80 & 257 & 337 & 0,51 \\
\hline Latvia & 66 & 215 & 281 & 0,43 \\
\hline Luxembourg & 56 & 181 & 237 & 0,36 \\
\hline Estonia & 47 & 152 & 199 & 0,30 \\
\hline Cyprus & 35 & 112 & 147 & 0,22 \\
\hline Malta & 17 & 54 & 71 & 0,11 \\
\hline
\end{tabular}

Fuente: Comisión Europea.

Para garantizar al máximo que todos los reubicados son candidatos claros a recibir el asilo, solo viajarán a otros Estados europeos los extranjeros cuyas solicitudes de asilo sean aceptadas al menos en un 75\% de los Estados. Datos recientes de Eurostat colocaban en este grupo a sirios y eritreos, aunque en los últimos meses también los iraquíes tienen esa elevada tasa de concesión de asilo. 
De la cuota global del primer año de 66.000 refugiados, a España le corresponden 6.127 de los refugiados llegados a Grecia y 1.896 de los llegados a Italia, es decir, un total de 8.023 refugiados.

En Enero de 2016, la Comisión Europea hizo públicas sus prioridades en la materia para este nuevo año y señaló que a lo largo de 2015 tan sólo se habían reubicado a 272 personas de las 160.000 incluidas en el acuerdo de Septiembre.

Este balance mostraba, a todas luces, la irresponsabilidad de los Estados miembros al incumplir de forma tan manifiesta los compromisos adquiridos y, también, por extensión, mostraba el fracaso de la actuación de las instituciones europeas.

\section{Reino Unido: nuevos pasos en la negociación con la UE}

En Noviembre, el primer ministro británico David Cameron pronunció un discurso en un laboratorio de ideas londinense, la Chatham House, en el que defendió la permanencia de su país en «una UE reformada» como la mejor manera de defender no solo la prosperidad económica, sino también la seguridad nacional del Reino Unido.

Inauguraba así «la fase formal» de la negociación, que dijo afrontar «con una visión más práctica que emocional; con la cabeza, no con el corazón». Expuso también las cuatro principales demandas que dijo trasladaría a sus socios europeos en los meses siguientes y que dejó por escrito en una carta remitida al presidente del Consejo Europeo, Donald Tusk:

- La puesta en marcha de mecanismos que aseguren la protección de los intereses de los países que permanecen fuera del euro frente a los avances en la integración de la Eurozona,

- Que la competitividad «esté grabada en el ADN de la Unión Europea», lo que implica «recortar las cargas impositivas a las empresas»,

- «Eximir al Reino Unido del principio de una Unión cada vez más estrecha» y «reforzar el papel de los Parlamentos nacionales», no solo con palabras cálidas sino mediante «reformas legales irreversibles»,

- «Afrontar los abusos del principio de libre circulación de personas» y permitir al Reino Unido controlar la migración procedente de la UE.

Este último punto implicaría que los inmigrantes europeos no podrían reclamar las ayudas públicas mientras estén buscando trabajo como son las prestaciones de desempleo o las compensaciones para los trabajadores con 
salarios más bajos en forma de, por ejemplo, créditos fiscales. «Si aquellos que vienen de la UE no han encontrado empleo en seis meses, podrán ser obligados a marcharse», explicó. «Y hemos propuesto», añadió, «que las personas que vienen a Reino Unido desde la UE deberán vivir y contribuir aquí durante cuatro años antes de tener derecho a solicitar ayudas».

\section{El Informe de los Cinco Presidentes sobre el futuro de la gobernanza económica europea}

El 22 de Junio de 2015 el grupo de los «cinco presidentes» (los de la Comisión Europea, Cumbre del Euro, Eurogrupo, Banco Central Europeo y Parlamento Europeo), hicieron público un ambicioso plan para profundizar en la Unión Económica y Monetaria, UEM a partir del 1 de julio de 2015, con el objetivo de completarla en 2025 a más tardar.

El informe plantea en su primer capítulo, titulado de forma significativa «Características de una Unión Económica y Monetaria profunda, auténtica y justa», la hoja de ruta a seguir en cuatro frentes:

- una «Unión Económica» auténtica que garantice que cada Economía disponga de las características estructurales que le permitan prosperar dentro de la Unión Monetaria,

- una «Unión Financiera» que garantice la integridad de nuestra moneda en la Unión Monetaria y aumente el reparto de riesgos con el sector privado,

- una «Unión Presupuestaria» que proporcione sostenibilidad y estabilización presupuestarias,

- y, por último, una «Unión Política» que siente las bases de las tres Uniones anteriores a través de un reforzamiento auténtico del control democrático y de la legitimidad de las instituciones.

El proceso de avance propuesto por los «cinco presidentes» se plantea según una secuencia de tres diferentes etapas:

- Etapa 1, «Profundización en la práctica» (del 1 de julio de 2015 al 30 de junio de 2017): utilizar los instrumentos existentes y los Tratados actuales para impulsar la competitividad y la convergencia estructural, poniendo en marcha políticas presupuestarias responsables a nivel nacional y de la zona del euro, para completar la Unión Financiera y mejorar la responsabilidad democrática.

En esta fase se plantean cuatro elementos prioritarios: la creación de «un sistema de autoridades de competitividad para la zona del euro», una aplicación reforzada del procedimiento de Desequilibrios Ma- 
croeconómicos, una mayor atención a los resultados en materia de empleo y en el ámbito social y una mayor coordinación de las políticas económicas en el marco de un Semestre Europeo renovado.

- Etapa 2, «Realización de la UEM»: se acordarían medidas concretas de mayor alcance para completar la estructura económica e institucional de la UEM. Específicamente, durante esta segunda etapa, el proceso de convergencia se haría más vinculante a través de una serie de parámetros de referencia para la convergencia establecidos de común acuerdo, a los que se podría dotar de naturaleza jurídica. El logro de progresos significativos hacia estos niveles, y el mantenimiento de los mismos una vez alcanzados, serían condiciones para que un Estado miembro de la zona del euro pudiera participar en un mecanismo de absorción de perturbaciones para la zona del euro durante esta segunda etapa.

- Etapa final (2025 a más tardar): una vez que todas las medidas estén plenamente vigentes, una profunda y auténtica UEM debería ser un área de estabilidad y prosperidad para todos los ciudadanos de los Estados miembros de la UE que comparten la moneda única. También debería ser atractiva para los otros Estados miembros de la UE que deseen formar parte de ella.

\section{Informes sobre la Ampliación de la UE}

El 10 de Noviembre, la Comisión hizo públicos los informes anuales sobre la Ampliación que pretenden evaluar la preparación de los diferentes países candidatos para cumplir los requisitos de adhesión a la UE ${ }^{1}$.

Respecto a Turquía, la Comisión aplaudía en sus informes el manejo de la crisis de refugiados sirios e iraquíes pero también criticaba con dureza la situación interna del país hasta el punto de poner en tela de juicio la garantía de los derechos humanos. «En el último año, deficiencias significativas

${ }^{1}$ La actual agenda de ampliación abarca a los países de los Balcanes Occidentales y a Turquía. Las negociaciones de adhesión con Turquía se iniciaron en 2005, pero avanzan muy lentamente. Con Montenegro hay negociaciones de adhesión en curso desde 2012 y con Serbia, desde 2014. El proceso de adhesión a la UE de la Antigua República Yugoslava de Macedonia, país candidato desde 2005, sigue en punto muerto. Albania obtuvo el estatuto de país candidato en 2014 y está abordando una serie de prioridades clave antes de que la Comisión pueda recomendar la apertura de las negociaciones de adhesión. En junio entró en vigor un Acuerdo de Estabilización y Asociación (AEA) con Bosnia y Herzegovina. Con Kosovo se firmó un Acuerdo similar en octubre de 2015. 
han afectado a la independencia de la justicia y a la libertad de reunión y de expresión en Turquía», señaló Hahn.

Bruselas también mostró su preocupación sobre el fin de las conversaciones de paz entre el Gobierno de Erdogan y el Partido de los Trabajadores Kurdos (PKK) y sobre la escasa y deteriorada separación de poderes, especialmente entre el ejecutivo y el judicial.

El comisario Hahn señaló que la crisis de los refugiados y las políticas económicas y democráticas han reforzado la cooperación entre el bloque de los aspirantes a adherirse al club comunitario y la propia UE, pero también destacó que aún existen importantes problemas en los siete países analizados, siendo la corrupción el denominador común en todos ellos.

«Pondremos nuestros esfuerzos en el Estado de derecho». Es un tema crucial, destacó el comisario, llevar a cabo reformas en el poder judicial para atajar el crimen organizado y la corrupción.

\section{Comisión: primera aplicación del Mecanismo del Estado de Derecho contra Polonia}

En Enero, la Comisión Europea decidió activar por primera vez un mecanismo legal que permitiría imponer sanciones a los países miembros que comprometan o amenacen el funcionamiento del Estado de Derecho, ante «violaciones graves y persistentes de los valores de la UE».

Según Bruselas, la actuación del nuevo Gobierno ultraconservador de Polonia en relación a la aprobación de dos normas sobre el Tribunal Constitucional y sobre los medios de comunicación públicos, justifican la aplicación de este marco.

En efecto, el llamado «mecanismo del Estado de Derecho» es un procedimiento a través del cual la Comisión puede denunciar eventuales riesgos o amenazas sobre las reglas democráticas y obliga al país a dar explicaciones. Y aunque es cierto que la capacidad ejecutiva de esa herramienta es limitada (si el Gobierno acusado se resiste a cambiar, solo queda despojarlo de sus derechos de voto en la UE, un recurso extremo que nunca se ha usado), Bruselas confía en que el mero inicio del proceso lleve a las autoridades polacas a reconsiderar las prácticas identificadas como una amenaza al Estado de Derecho.

Las amenazas que la Comisión considera como posibles vulneraciones del Estado de Derecho son la norma que asegura al gobierno polaco el control de los medios de comunicación públicos y, especialmente, la legislación que resta margen de maniobra al Tribunal Constitucional. Bruselas considera, en efecto, que la independencia de esta Corte de Justicia 
y de los medios públicos son dos pilares básicos en cualquier Estado de Derecho.

\section{Cuestiones generales de la actualidad económica}

\section{BCE: Ampliación de la compra masiva de Deuda pública}

En Diciembre, el BCE anunció la ampliación del programa de compras de deuda pública y privada a gran escala, conocido como «Flexibilización Cuantitativa»(QE, Quantitative Easing en inglés) hasta, al menos, Marzo de 2017, seis meses más, con lo que la cifra global de compra de deuda llegará, previsiblemente, a 1,5 billones de euros.

En el lanzamiento del programa, el presidente del BCE señalaba que el objetivo debería ser conseguir un «rebote de la inflación» y reducir las primas de riesgo, es decir, los tipos de interés que pagan los países por su deuda en términos de diferencial respecto los intereses de los bonos alemanes. Y, también, dijo confiar en que el programa mejorase las condiciones de financiación de empresas y particulares.

La intervención del BCE se está realizando a través de los Bancos Centrales de los países de la Eurozona. Desde Marzo de 2015, están comprando bonos con una calificación mínima BBB en los mercados secundarios, aunque con la condición, impuesta por Alemania, de que únicamente el 20\% del riesgo de las compras se mutualiza. De esta forma, la mayor parte de las posibles pérdidas o ganancias que se produzcan se integrarán directamente en los balances de cada uno de los Bancos Centrales nacionales.

Por otra parte, a lo largo de este segundo semestre de 2015 los tipos de interés aplicables a las operaciones principales del BCE se han mantenido en los niveles fijados en Septiembre de 2014, salvo los relativos a las facilidades de depósito que en Diciembre fueron rebajados de $-0,20 \%$ a $-0,30 \%$. Con esta medida, el BCE cobra más intereses a los bancos que prefieren depositar sus fondos en el propio BCE, en lugar de utilizarlos en préstamos para empresas y hogares.

\section{Acuerdo Trasatlántico UE-EEUU: nuevas propuestas para el arbitraje en materia de inversiones}

La creación de un Tribunal de arbitraje que dilucide las controversias derivadas de las inversiones transatlánticas es uno de los aspectos más controvertidos del Tratado, ya que sus decisiones podrían capacitar a las multi- 
nacionales americanas para recurrir y revocar leyes estatales europeas justificándose en la protección de sus inversiones.

La Comisión ha ido planteando a lo largo del año diferentes alternativas para este Tribunal. En un principio propuso que los miembros del Tribunal serían personas libres de conflictos de interés y con cualificación jurídica en sus países, para evitar que las mismas personas actúen como jueces en un arbitraje y como abogados en otros, o que, tras mediar en un conflicto acaben posteriormente en el consejo de administración o a sueldo de la empresa sobre la que tomaron una decisión. Posteriormente también se planteó la posibilidad de establecer un Tribunal de apelación permanente con capacidad de confirmar o revocar el resultado del arbitraje.

Y finalmente, en Septiembre, la comisaria Cecilia Malmström propuso la sustitución del mecanismo extrajudicial de árbitros privados por un Tribunal de Inversión, al que podrían recurrir las empresas europeas y estadounidenses que se vieran perjudicadas por determinadas decisiones adoptadas en cualquiera de los dos bloques.

Según esta nueva propuesta, el sistema consistiría en dos tribunales: un Tribunal de primera instancia, constituido por 15 jueces (cinco europeos, cinco estadounidenses y cinco de terceros países) y otro Tribunal de apelación, con dos jueces europeos, dos estadounidenses y dos de otras nacionalidades. La idea sería que este Tribunal, responsable inicialmente de los casos más relevantes sobre expropiaciones, nacionalizaciones y conflictos similares, se consolide para resolver litigios de inversión derivados de otros acuerdos internacionales que la UE pueda firmar en el futuro, especialmente con países «con sistemas judiciales no muy sólidos», según precisó Malmström. Este nuevo instrumento estaría, en palabras de la responsable de Comercio de la Comisión, «sujeto a principios democráticos y escrutinio público».

El objetivo de la iniciativa sería que, con el tiempo, el futuro «Tribunal Internacional de Inversiones» sustituya a todos los mecanismos de solución de diferencias previstos en los acuerdos de la UE, en los acuerdos de los Estados miembros de la UE con terceros países y en los acuerdos comerciales y de inversión celebrados entre países no pertenecientes a la UE. Su mayor ámbito de actividad incrementaría su eficacia y legitimidad en la solución internacional de diferencias en materia de inversión.

Según fuentes expertas, será particularmente difícil la aceptación de esta propuesta por parte de los socios estadounidenses, ya que este país no vería con buenos ojos la existencia de un cuerpo público ajeno a su jurisdicción que sentencie sobre decisiones adoptadas por sus empresas o sus autoridades públicas. 


\section{La Unión de los Mercados de Capitales}

La Comisión Europea publicó en Septiembre su plan de acción para crear la «Unión de los Mercados de Capitales», UMC, entre los 28 Estados miembros de la UE.

Bruselas destacaba que en otras partes del mundo está más extendido el recurso a otras fuentes de financiación, complementarias a la financiación bancaria, como el capital riesgo o la microfinanciación colectiva y que, en este sentido, la mayor variedad de fuentes de financiación sería positiva para las inversiones y para la estabilidad financiera en la UE, al mitigar los efectos que pueden provocar los eventuales problemas en el sector bancario. clave:

El plan de acción se estructura en torno a los siguientes principios

- Crear más oportunidades para los inversores,

- Conectar financiación y economía real,

- Fomentar un sistema financiero más sólido y resiliente,

- Profundizar la integración financiera y aumentar la competencia.

Y aunque se trata de un proyecto a medio plazo, la Comisión anunciaba el lanzamiento de dos paquetes de propuestas legislativas, destinadas a reactivar la titulización de alta calidad y a fomentar la inversión a largo plazo en infraestructuras:

- Las primeras propuestas pretenden crear un marco reglamentario para las titulizaciones. simple, transparente y normalizado, y sujeto a un control de supervisión. Las titulizaciones son procesos de creación de instrumentos financieros que reagrupan activos (por ejemplo, préstamos a empresas o a particulares) y que los inversores pueden comprar.

- La segunda propuesta pretende eliminar los obstáculos prudenciales que impiden a las entidades aseguradoras desempeñar un papel importante en los proyectos europeos de infraestructuras, creando una categoría distinta de activos de infraestructuras y reduciendo el importe de capital que las entidades aseguradoras deben mantener con respecto a la deuda y el capital de los proyectos de infraestructuras subvencionables.

Junto con estas iniciativas, la Comisión también lanzaba dos procesos de consultas, que darán pie a nuevas acciones en 2016 y 2017.

La primera consulta hace referencia a los fondos de capital riesgo y de emprendimiento social, ya que Bruselas pretende conocer las opiniones sobre si la introducción de cambios en la normativa comunitaria pu- 
diera impulsar la utilización de estos fondos (modificaciones en temas como las restricciones aplicables a los gestores de los fondos, el nivel de inversión mínima para los inversores o la posibilidad de gestores de terceros países).

La segunda consulta se refiere a los bonos garantizados y se centra en la creación de un marco paneuropeo que combata la actual fragmentación del mercado europeo, una situación que dificulta la normalización de las prácticas de suscripción y declaración y que obstaculiza la profundización, liquidez y accesibilidad de los mercados.

A más largo plazo, en 2019, la Comisión acometerá los cambios necesarios para afrontar algunas de las barreras más difíciles y duraderas a la mejor ubicación de los capitales en toda Europa. Con la UMC la Comisión también prevé eliminar los obstáculos a las inversiones transfronterizas en la UE, de modo que las empresas y los proyectos de infraestructuras puedan financiarse más fácilmente con independencia del lugar en que estén ubicados.

\section{Unión Bancaria: Sistema Europeo de Garantía de Depósitos Bancarios}

A finales de Noviembre La Comisión hizo pública su propuesta para crear un Sistema único de Garantía de los Depósitos bancarios para toda la zona del euro.

Se prevén tres fases para su implantación:

Fase 1: Reaseguro (hasta 2020): En esta etapa, los sistemas nacionales de garantía de depósitos solo podrán acceder a los fondos del sistema europeo cuando hayan agotado sus recursos propios; y, como en las demás fases, si se ajustan a la Directiva sobre el sistema de garantía de depósitos.

Los fondos del sistema europeo de garantía de depósitos proporcionarán fondos adicionales a los sistemas nacionales, pero solo hasta un determinado nivel, de manera que existirán salvaguardias para garantizar que, en efecto, los sistemas nacionales accedan al sistema europeo solo cuando esté justificado. Cualquier uso que se haga de los fondos del sistema será objeto de un estrecho seguimiento.

Fase 2: Coaseguro (2020-2024): En 2020 el sistema europeo de garantía de depósitos se convertirá en un sistema progresivamente mutualizado («coaseguro»), aunque sujeto a límites y salvaguardias adecuados contra los abusos. La diferencia fundamental en esta fase sería que los sistemas nacionales no estarán obligados a agotar sus fondos propios antes de acceder a los fondos del sistema europeo de garantía de depósitos.

El sistema europeo de garantía de depósitos estará en disposición de contribuir a una parte de los costes a partir del momento en que haya que 
reembolsar a los depositantes. Esto introduce un mayor grado de distribución de riesgo entre los regímenes nacionales a través del sistema europeo de garantía de depósitos. La cuota contributiva del sistema europeo de garantía de depósitos comenzará a un nivel relativamente bajo (20 \%), y se incrementará en un período de cuatro años.

Fase 3: Seguro pleno (2024): Al incrementar gradualmente la cuota de riesgo que el sistema europeo de garantía de depósitos asume al $100 \%$, dicho sistema aseguraría plenamente los sistemas nacionales de garantía de depósitos a partir de 2024.

El Fondo Europeo de Garantía de Depósitos se creará, por tanto, desde el primer momento, financiándose mediante las contribuciones de los bancos, ajustadas en función del riesgo, lo que significa que los bancos con más riesgos efectuarán aportaciones más elevadas, y su gestión se confiará a la Junta Única de Resolución del Mecanismo Único de Resolución.

El sistema, que significa que los riesgos del sector financiero serán gradualmente compartidos, es una propuesta esencial para completar la Unión Bancaria, en la medida en que afianzará la protección de los depositantes bancarios, reforzará la estabilidad financiera y reducirá aún más el vínculo entre los bancos y las deudas soberanas.

\section{Tribunal de Justicia: nueva sentencia contra la Ley Hipotecaria española}

En Octubre, el Tribunal de Justicia europeo emitió un nuevo dictamen sobre la Ley Hipotecaria española. Como en ocasiones anteriores, se trataba de una petición de decisión prejudicial, planteada esta vez por un Juzgado de Primera Instancia de Martorell (Barcelona), para resolver un litigio que enfrentaba al banco BBVA y a tres consumidores que formularon su oposición a una disposición transitoria, introducida en la legislación respecto al procedimiento de ejecución hipotecaria como consecuencia de una sentencia anterior del Tribunal comunitario de 2013.

Estos consumidores, inmersos en procedimientos de ejecución hipotecaria en curso cuando entró en vigor la nueva Ley, alegaban que el plazo de oposición de un mes incluido en esta disposición era del todo insuficiente para hacer valer sus derechos contra las eventuales cláusulas abusivas del contrato hipotecario. Y denunciaban asimismo el hecho de que este plazo comenzara a contabilizarse a partir de la publicación de la Ley en el Boletín Oficial español y no a partir del momento en que se produjera una notificación individualizada, ya que esta disposición dificultaba sobremanera el ejercicio del derecho de oposición. 
La sentencia dictada en Octubre declaraba que el plazo «preclusivo» de un mes para formular una oposición parece, en principio, materialmente suficiente para preparar e interponer un recurso judicial efectivo, de modo que resulta razonable y proporcionado atendiendo a los derechos e intereses de que se trata. Sin embargo, los jueces europeos señalaban que «el mecanismo establecido por el legislador para iniciar el cómputo del plazo, esto es, la publicación de la Ley en el Boletín Oficial del Estado, es contrario al principio de efectividad».

La disposición transitoria que establece que el plazo comience a contarse sin que los consumidores afectados sean informados personalmente de la posibilidad de alegar un nuevo motivo de oposición en el marco de un procedimiento de ejecución ya iniciado antes de la entrada en vigor de la nueva Ley, no garantiza que se pueda aprovechar plenamente ese plazo y, en consecuencia, no garantiza el ejercicio efectivo del nuevo derecho reconocido por la modificación legislativa española.

El Tribunal de Justicia dictaminaba, en este sentido, que «existe un elevado riesgo de que el plazo expire sin que los consumidores afectados puedan hacer valer de forma efectiva y útil sus derechos por la vía judicial», lo que supone el incumplimiento de la Directiva 93/13/CEE del Consejo, de 5 de abril de 1993, sobre las cláusulas abusivas en los contratos celebrados con consumidores.

\section{Comisión: dictamen contra las cláusulas suelo en las hipotecas españolas}

La Comisión Europea emitió en Octubre un informe contrario a la sentencia del Tribunal Supremo español que había obligado a los bancos españoles a devolver las cláusulas suelo de las hipotecas (mínimo a pagar en las cuotas de la hipoteca aunque los tipos acordados con la entidad sean inferiores) solo a partir del 9 de mayo de 2013, fecha de la sentencia, sin exigir por tanto la retroactividad sobre las sumas cobradas con anterioridad a esta fecha.

Por el contrario, Bruselas consideraba que debe reintegrarse todo el dinero cobrado por aplicar estas cláusulas desde el inicio del crédito, y no solo desde mayo de 2013, desmontando así la argumentación del alto Tribunal español que limitaba la retroactividad.

El informe de la Comisión es una de las alegaciones presentadas ante el Tribunal de Justicia Europeo, quien deberá emitir una sentencia próximamente; si, en efecto, los jueces siguen el razonamiento de la Comisión, podría suponer una factura multimillonaria para la banca española. 


\section{Competencia: ilegalidad de los pactos fiscales con multinacionales}

En Octubre, la comisaria de Competencia, Margrethe Vestager, inició una acción contra los acuerdos fiscales abusivos que algunos países de la UE suscriben con grandes multinacionales para ofrecerles una drástica rebaja de impuestos, en detrimento de los ingresos fiscales del resto de Estados miembros de la Unión.

Holanda y Luxemburgo fueron los dos primeros países en recibir las críticas de la Comisión por sus acuerdos con Starbucks en 2008 y con Fiat Finance en 2012 respectivamente.

Bruselas calcula que ambas compañías, cuyo Impuesto de Sociedades en esos países no llegaba al millón de euros, han ahorrado entre 20 y 30 millones.

La reacción del Ejecutivo de Luxemburgo llegó a través de su ministro de Finanzas, Pierre Gramegna, quien señaló que «la UE ha utilizado argumentos sin precedentes para concluir que hay una ayuda de Estado». «Discrepamos de la Comisión y nos reservamos todos nuestros derechos», añadió, lo que anticipaba un probable recurso ante el Tribunal de Justicia en la UE.

Este posible recurso, sin embargo, no paraliza la ejecución del dictamen comunitario que exigía a Holanda y Luxemburgo la recuperación de las ayudas ilegales recibidas por Starbucks y Fiat en forma de rebaja de su Impuesto de Sociedades.

Y en Diciembre, la Comisión también hizo pública su acusación al gigante norteamericano de comida rápida, McDonald's, por no pagar el Impuesto de Sociedades correspondientes a los cánones cobrados a sus restaurantes en la UE y Rusia desde 2009.

Bruselas abría una «investigación exhaustiva» sobre el trato fiscal dispensado a la compañía en Luxemburgo. Según estas fuentes comunitarias, McDonald's no habría abonado en los seis últimos años ni un euro en concepto de Impuesto de Sociedades en el Gran Ducado por los cánones que abonan los restaurantes franquiciados en todo el territorio de la UE y en Rusia por el derecho de utilizar su marca, a pesar de haber obtenido cuantiosos beneficios (250 millones de euros sólo en 2013).

En 2009 y en virtud de la norma que evita la doble imposición, la Comisión había concluido que la filial europea de McDonald's no debía pagar el Impuesto en Luxemburgo porque «los beneficios estaban sujetos a la imposición de EE UU», aunque la empresa debía demostrar anualmente que los cánones transferidos a EE UU se habían declarado y que quedaban sujetos a imposición en dicho país. El ejecutivo comunitario opina, ahora, que tampoco tributó en EE UU. 
La comisaria Vestager remarcó que una resolución fiscal que acepte que la empresa no pague ningún impuesto, ni en Europa ni en EE UU, «debe examinarse cuidadosamente con arreglo a las normas comunitarias sobre ayudas estatales». «La finalidad de los convenios en materia de doble imposición es evitar la doble imposición, no justificar la doble no imposición», señaló.

La Comisión pretende averiguar si las autoridades luxemburguesas derogaron «de forma selectiva» las disposiciones que afectan a la doble imposición, concediendo así a McDonald's una ventaja de la que no gozan sus competidores.

Por otra parte, en Enero la comisaria Vestager también anunció la finalización de una de las investigaciones abiertas por su departamento al Gobierno belga por irregularidades en su sistema de incentivos fiscales.

Según Bruselas, el sistema de pactos fiscales («Tax Rulings») belga era ilegal, por lo que el gobierno de este país tendrá que recuperar 700 millones de euros de hasta 35 multinacionales que se beneficiarios de esas ayudas ilegales de Estado.

La comisaria explicó que estas multinacionales, casi todas europeas, se beneficiaron de este esquema desde el año 2005, logrando pagar «sustancialmente menos impuestos de los que les correspondería» por sus beneficios.

El esquema reducía la base imponible entre un 50 y un $90 \%$, gracias a una cláusula de «beneficios excesivos» que permitía deducciones por pertenecer a un grupo multinacional. Según Vestager, «Bélgica ha dado a un número selectivo de multinacionales sustanciales ventajas fiscales, haciendo que rivales más pequeños que no son multinacionales no pudieran beneficiarse de las mismas oportunidades».

\section{Telefonía móvil: eliminación de las tarifas de itinerancia en 2017}

En la votación del pleno de Octubre, el Parlamento Europeo dio su aprobación a la prohibición total de costes suplementarios por utilizar el teléfono móvil en el extranjero dentro de la UE a partir de Junio de 2017.

Como paso previo a esta prohibición, a partir del 30 de abril de 2016 los sobrecostes por itinerancia (que se suman al precio facturado en el país de procedencia) no podrán rebasar 0,05 euros por minuto para las llamadas salientes, 0,02 euros por mensajes de texto SMS, o 0,05 euros por megabyte de uso de internet móvil.

La normativa aprobada también obligará a las compañías que ofrecen acceso a internet a tratar todo el tráfico de manera equitativa, lo que significa que no podrán bloquear o ralentizar deliberadamente la descarga $\mathrm{o}$ 
subida de contenidos, las aplicaciones o los servicios de operadores o receptores concretos, excepto en caso de orden judicial para garantizar el cumplimiento de la ley, evitar la congestión en la red o combatir ataques cibernéticos. De resultar necesarias, estas medidas de gestión del tráfico, tendrán que ser «transparentes, no discriminatorias y proporcionadas» y limitadas en el tiempo.

«Gracias a este acuerdo, Europa se convertirá en la única región del mundo que garantizará por ley un internet abierto y neutral. El principio de la neutralidad será de aplicación directa en los 28 Estados miembros. No habrá un internet de dos velocidades», recalcó la eurodiputada Pilar del Castillo.

\section{Tribunal de Justicia anulación de la decisión de la Comisión que declaraba ilegal el Tax Lease del sector naval español}

En Diciembre, el Tribunal General de la UE anuló la Decisión de la Comisión Europea según la cual el denominado «sistema español de arrendamiento fiscal», SEAF, constituía una ayuda estatal ilegal.

Este sistema, conocido como «Tax Lease» permitía generar créditos fiscales a las empresas que invertían en la construcción de buques para su posterior arrendamiento y venta a navieras que, a su vez, los compraban con un descuento de entre el $20 \%$ y el $30 \%$. Las empresas rebajaban su factura fiscal, las navieras adquirían barcos más baratos y los astilleros recibían pedidos y generaban empleo.

En Julio de 2013 el ejecutivo comunitario declaró el «Tax Lease» ilegal y su responsable, el entonces comisario de Competencia Joaquín Almunia, exigió la devolución de las ayudas conseguidas entre 2007 y 2011 por parte de los inversores.

Pero ahora, dos años después, el Tribunal comunitario ha considerado que la conclusión de Bruselas no está «suficientemente motivada» como para probar que el sistema podía falsear la competencia y afectar a los intercambios comerciales entre Estados miembros.

La sentencia señalaba que, en efecto, la competencia no fue atacada puesto que «cualquier operador podía beneficiarse de las ventajas fiscales en cuestión efectuando cierto tipo de operaciones que cualquier empresa sin distinción podía realizar en idénticas condiciones». Las ayudas económicas no eran, por tanto, selectivas.

Tras la decisión del Tribunal, la Comisión dispone de dos meses para interponer un recurso de casación. 


\section{Derechos de autor (Copyright)}

Los derechos de autor de esta publicación pertenecen a la editorial Universidad de Deusto. El acceso al contenido digital de cualquier número de Cuadernos Europeos de Deusto (CED) es gratuito, transcurridos 6 meses desde su publicación. Los trabajos podrán descargarse, copiar y difundir, sin fines comerciales y según lo previsto por la ley. Así mismo, los trabajos editados en CED pueden ser publicados con posterioridad en otros medios o revistas, siempre que el autor indique con claridad y en la primera nota a pie de página que el trabajo se publicó por primera vez en CED, con indicación del número, año, páginas y DOI (si procede). 